\title{
REDUCTIVITY AND THE AUTOMORPHISM GROUP OF LOCALLY COMPACT GROUPS
}

\author{
BY
}

\author{
DONG HOON LEE
}

\begin{abstract}
In this paper, we study reductivity of locally compact groups and its effect on the automorphism group and generalize the classical results on the automorphism group of analytic semisimple groups on the one hand and of compact groups on the other.
\end{abstract}

1. Introduction. A locally compact group $G$ is said to be reductive if the first cohomology group $H^{1}(G, V)=0$ for any continuous finite-dimensional real $G$ module $V$. Here the cohomology group is that of Eilenberg-Mac Lane based on the continuous cochains. As we shall see later, this is equivalent to the statement that every finite-dimensional real $G$-module is semisimple. In this sense, a compact group is reductive and a semisimple analytic group is also reductive. In his early work [7], Iwasawa proved that if $G$ is a compact group, then the identity component of the automorphism group of $G$ consists of the inner automorphisms induced by the elements of the identity component of $G$. In this work, we study the reductivity of locally compact groups and its effect on their automorphism groups. More specifically,we are mainly concerned with establishing Iwasawa's result for reductive groups. In $\$ 2$, we examine some of the properties of reductive groups. For example, a characterization of compactly generated reductive groups in terms of conjugacy classes of homomorphisms into Lie groups is given (Theorem 1). In \$3, we investigate basic structures of connected reductive groups for later use, and $\$ 4$ establishes Iwasawa's result for any connected reductive groups. Finally, in $\$ 5$ we generalize Iwasawa's result to a locally compact group which is an extension of a semisimple group by a compact group.

Throughout this paper, we adopt the following notation. For any topological group $G, G_{0}$ will denote the identity component of $G$ and $Z(G)$ will denote the center of $G$.

2. Reductivity of locally compact groups.

(2.1) Let $G$ be a locally compact group. By a continuous $G$-module, we mean a Hausdorff topological vector space $V$ over the field $R$ of real numbers together

Received by the editors March 25, 1975.

AMS (MOS) subject classifications (1970). Primary 22D12, 22D45, 22E15, 22 Co5.

Key words and phrases. Reductivity, cochains, semisimple, conjugacy classes, orbits, radical, adjoint representation. 
with a $G$-module structure such that the corresponding map $(x, v) \rightarrow x \cdot v: G \times$ $V \rightarrow V$ is continuous, and the map $v \rightarrow x \cdot v: V \rightarrow V$ is a linear automorphism of $V$, for every $x \in G$.

A continuous $G$-module $V$ is called semisimple if every submodule is a direct $G$-module summand of $V$.

For two $G$-modules $V$ and $W$, the topological vector space $\operatorname{Hom}_{R}(V, W)$ can be made into a continuous $G$-module, if we define the action of $G$ on $\operatorname{Hom}_{R}(V, W)$ by $(x \cdot f)(v)=x f\left(x^{-1} \cdot v\right)$ for all $x \in G, f \in \operatorname{Hom}_{R}(V, W)$ and $v \in V$. If we denote by $V^{G}$ the subspace of a $G$-space $V$ consisting of all the elements $v \in V$ with $x \cdot v=v$ for all $x \in G$, then $\operatorname{Hom}_{R}(V, W)^{G}$ consists of all the $R$-linear maps commuting with the action of $G$.

(2.2) A locally compact group $G$ is called reductive if $H^{1}(G, V)=0$ for every finite-dimensional continuous $G$-module. Here the cohomology group is that of Eilenberg-Mac Lane based on continuous cochains. For a detailed discussion, see Mostow [11]. These cohomology groups reduce to the Van Est cohomology groups when $G$ is a Lie group and to the Eilenberg-Mac Lane groups when $G$ is discrete. For any continuous $G$-module $V$, we may compute $H^{p}(G, V)$ from the complex of the nonhomogeneous cochains for $G$ in $V$. Thus $H^{0}(G, V)$ may be identified with $V^{G}$, and $H^{1}(G, V)$ is isomorphic, as a topological vector space, with the quotient space of the space $Z^{1}(G, V)$ of all continuous crossed homomorphisms of $G$ into $V$ (topologized by the compact-open topology) modulo the subspace $B^{1}(G, V)$ consisting of the maps of the form $x \rightarrow x v-v$, where $v \in V$.

(2.3) The following justifies our term "reductivity."

Proposition 1. Let $G$ be a locally compact group. Then $G$ is reductive if and only if every finite-dimensional continuous G-module is semisimple.

Proof. Let $G$ be reductive, and let $0 \rightarrow V \rightarrow W \stackrel{\pi}{\rightarrow} U \rightarrow 0$ be an exact sequence of finite-dimensional continuous $G$-modules. We prove that this sequence splits as $G$-modules. To do this, we consider the exact sequence of $G$ modules

$$
0 \rightarrow \operatorname{Hom}_{R}(U, V) \rightarrow \operatorname{Hom}_{R}(U, W) \stackrel{\pi_{*}}{\longrightarrow} \operatorname{Hom}_{R}(U, U) \rightarrow 0
$$

(see (2.1)). This short exact sequence induces the cohomology exact sequence

$$
\begin{aligned}
0 \rightarrow H^{0}\left(G, \operatorname{Hom}_{R}(U, V)\right) & \rightarrow H^{0}\left(G, \operatorname{Hom}_{R}(U, W)\right) \\
& \rightarrow H^{0}\left(G, \operatorname{Hom}_{R}(U, U) \rightarrow H^{1}\left(G, \operatorname{Hom}_{R}(U, V)\right) \rightarrow \cdots .\right.
\end{aligned}
$$

By hypothesis, $H^{1}\left(G, \operatorname{Hom}_{R}(U, V)\right)=0$ while $H^{0}\left(G, \operatorname{Hom}_{R}(U, W)\right)=$ $\operatorname{Hom}_{R}(U, W)^{G}$, etc. Thus $\operatorname{Hom}_{R}(U, W)^{G} \stackrel{\pi^{*}}{\longrightarrow} \operatorname{Hom}_{R}(U, U)^{G}$ is surjective, and 
if we choose $\sigma \in \operatorname{Hom}_{R}(U, W)^{G}$ such that $\pi^{*}(\sigma)=1_{U}$, then $\pi \cdot \sigma=1_{U}$, proving the assertion.

Now assume that $G$ is a locally compact group such that every finite-dimensional continuous $G$-module is semisimple. We first note that any continuous homomorphism of $G$ into $R$ is trivial. This follows from the simple fact that there is a 2-dimensional space on which $R$ acts unipotently and that this together with a nontrivial homomorphism into $R$ would give a nonsemisimple $G$-module.

Now to prove the reductivity of $G$, let $f \in Z^{1}(G, V)$, where $V$ is a finitedimensional continuous $G$-module. Let $W=V \oplus R$ and we make $W$ into a continuous $G$-module by defining the action of $G$ on $W$ by $x \cdot(v, \lambda)=(x \cdot v+$ $\lambda(f(x)), \lambda)$ for $x \in G$ and $(v, \lambda) \in W$. Since $V \cong V+\{0\}$ is a $G$-submodule of $W$ of codimension 1 , semisimplicity of the $G$-module $W$ implies that there exists a $G$-fixed element $(v, \lambda) \in W$ with $\lambda \neq 0$. Letting $v_{0}=-\lambda^{-1} v$, we see that $\left(v_{0},-1\right)=x\left(v_{0},-1\right)=\left(x \cdot v_{0}-f(x), 1\right)$, proving that $f(x)=x v_{0}-v_{0}$. Hence $f \in B^{1}(G, V)$.

(2.4) Below we give a different characterization of a reductive group when it is compactly generated.

Let $G$ and $H$ be locally compact groups, and let $\operatorname{Hom}(G, H)$ denote the space of all continuous homomorphisms of $G$ into $H$ topologized by the compact open topology. Then $H$ acts on $\operatorname{Hom}(G, H)$ by conjugation. That is, $(x \cdot f)(y)$ $=x f(y) x^{-1}$ for $x \in H, y \in G$ and $f \in \operatorname{Hom}(G, H)$.

THEOREM 1. Let $G$ be a compactly generated locally compact group. Then the following are equivalent.

(i) $G$ is reductive.

(ii) For any Lie group $H$, the connected components of the space $\operatorname{Hom}(G, H)$ are exactly the orbits of $H_{0}$ in $\operatorname{Hom}(G, H)$.

Proof. (i) implies (ii). Let $H$ be a Lie group with Lie aglebra $H$ and let $C$ be a connected component of $\operatorname{Hom}(G, H)$. If $f \in C$, then we show that $C$ is equal to the orbit $H_{0} \cdot f$ of $H_{0}$ at $f$. Let Ad denote the adjoint representation of $H$. Then $\operatorname{Ad} \cdot f$ is a continuous representation of $G$ in $H$. Since $H^{1}(G, H)=$ 0 , it follows from the result in [8, Theorem 2, p. 359] that $H_{0} \cdot f$ is an open neighborhood in $\operatorname{Hom}(G, H)$ and is contained in $C$. To show that $H_{0} \cdot f$ is also

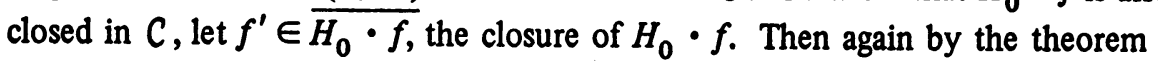
cited above, $H_{0} \cdot f^{\prime}$ is an open neighborhood of $f^{\prime}$. Hence $H_{0} \cdot f \cap H_{0} \cdot f^{\prime} \neq$ $\varnothing$ and consequently $H_{0} \cdot f^{\prime}=H_{0} \cdot f$, proving that $f^{\prime} \in H_{0} \cdot f$ and $H_{0} \cdot f=$ $\overline{H_{0} \cdot f}$ follows. Since $C$ is connected, $C=H_{0} \cdot f$, proving (ii).

(ii) implies (i). Let $V$ be a finite-dimensional continuous $G$-module, and let $\rho: G \rightarrow \mathrm{GL}(V)$ be the continuous representation of $G$ in $V$ which is associated with the $G$-module structure on $V$. Regarding $V$ as an additive group, we form 
the semidirect product $V \cdot \mathrm{GL}(V)$ with respect to the natural action of GL(V) on $V$. Then $V \cdot \operatorname{GL}(V)$ is a Lie group. For each $f \in Z^{1}(G, V)$, we define $f^{\prime}$ : $G \rightarrow V \cdot G L(V)$ by $f^{\prime}(x)=(f(x), \rho(x)), x \in G$. Then clearly $f^{\prime}$ is a continuous homomorphism. We define $\varphi: Z^{1}(G, V) \rightarrow \operatorname{Hom}(G, V \cdot \operatorname{GL}(V))$ by $\varphi(f)=$ $f^{\prime}$. Then it is easy to see that $\varphi$ is continuous and, since $Z^{1}(G, V)$ is connected as a topological vector space, $\operatorname{Im}(\varphi)$ is contained in the connected component of $\operatorname{Hom}(G, V \cdot G L(V))$ which contains the homomorphism $x \rightarrow(0, \rho(x)), x \in G$. By (ii), this component is the orbit of $H_{0}$ at the homomorphism above. Hence there exists $\left(v_{0}, \theta\right) \in V \cdot \mathrm{GL}(V)$ such that, for every $f \in Z^{1}(G, V)$, we have

$$
\begin{aligned}
(f(x), \rho(x)) & =f^{\prime}(x)=\left(v_{0}, \theta\right)(0, \rho(x))\left(v_{0}, \rho\right)^{-1} \\
& =\left(v_{0}-\theta \cdot \rho(x) \theta^{-1}\left(v_{0}\right), \theta \cdot \rho(x) \cdot \theta^{-1}\right) \quad \text { for all } x \in G .
\end{aligned}
$$

Thus $f(x)=v_{0}-\rho(x) v_{0}$, proving that $f \in B^{1}(G, V)$ and $H^{1}(G, V)=0$ follows.

(2.5) It is clear that a quotient group of a reductive group is reductive. Also we have the following result.

Proposition 2. Let $K$ be a closed normal subgroup of a locally compact group $G$. If $K$ and $G / K$ are both reductive, then so is $G$. If $G$ is reductive and if $K$ is of finite index in $G$, then $K$ is reductive.

A proof of this proposition may be found in [6] in the equivalent form given in Proposition 1 in (2.3). In fact, the first assertion is Theorem 3.2 on p. 508 , and the second assertion follows directly from Theorem 3.3 on p. 509 and the fact that if $K$ is a normal subgroup of $G$, then every semisimple $G$-module is also semisimple as a $K$-module (Chevalley [1, Proposition 1, p. 262]).

As an easy consequence, we have

COROLLARY. Let $G$ be a locally compact group such that $G / G_{0}$ is compact. Then $G$ is reductive if and only if $G_{0}$ is reductive.

Proof. Suppose that $G_{0}$ is reductive. Since $G / G_{0}$ is compact, it is reductive. Hence by Proposition 2, $G$ is reductive. Conversely assume that $G$ is reductive. Since $G / G_{0}$ is compact, we can find a compact normal subgroup $K$ of $G$ such that $G / K$ is a Lie group. By the compactness of $K$, it becomes clear that the subgroup $G_{0} K / K$ is the identity component of $G / K$ and that $G / K$ is finite modulo $G_{0} K / K$. Hence $G_{0} K / K$ is reductive by Proposition 2. Now $G_{0}$ is an extension of the compact normal subgroup $K \cap G_{0}$ by $G_{0} / K \cap G_{0}$ and $G_{0} / K \cap G_{0} \cong G_{0} K / K$. Hence, by Proposition 2 again, $G_{0}$ is reductive.

3. The structure of connected reductive groups.

(3.1) Let $G$ be a connected locally compact group and let $R(G)$ denote the radical of $G$ (that is, the largest connected solvable normal subgroup of $G$; see 
[7]). If $R(G)=\{1\}$, then we say that $G$ is semisimple. It is easy to see that $G / R(G)$ is semisimple. Every semisimple group $G$ is a projective limit of semisimple analytic groups and hence its commutator subgroup is dense in $G$. We also remark that a semisimple group is reductive as an extension of a compact group by a semisimple Lie group (see Proposition 2).

(3.2) LEMMA. Let A be a totally disconnected normal (hence central) closed subgroup of a connected locally compact semisimple group $G$. If $G / A$ is a Lie group, then so is $G$.

Proof. We note that the lemma is proved in [3, Lemma 2, p. 426] for compact totally disconnected $A$. Let $K$ be a compact normal subgroup of $G$ so that $G / K$ is a Lie group. Then there is a canonical continuous isomorphsim $G / A \cap K$ $\rightarrow(G / A) \times(G / K)$ which is an isomorphism of topological groups. Thus $G / A \cap K$ is a Lie group. Since $A \cap K$ is compact, $G$ itself is a Lie group by what we have noted in the beginning of our proof.

(3.3) Proposition 3. Let $G$ and $H$ be connected semisimple locally compact groups and let $\varphi: H \rightarrow G$ be a dense embedding. Then $\varphi$ is an isomorphism.

Proof. Assume that $H$ and $G$ are both Lie groups. Then the result follows from a theorem of Van Est [12, Theorem 2.2.1, p. 324]. Assume now that $H$ is a Lie group. Then $G$ is necessarily a Lie group. To see this, we note first that $Z(G) \cap H=Z(H)$. Hence $\varphi$ induces a dense embedding $\hat{\varphi}: H / Z(H) \rightarrow$ $G / Z(G)$. Since $H / Z(H)$ is a semisimple Lie group, its inner automorphism group is closed in the automorphism group of $H / Z(H)$. Observing that $H / Z(H)$ has the trivial center, we may apply Theorem 1 of $[4, p .161]$ to conclude that $\hat{\varphi}$ is an isomorphism. In particular, $G / Z(G)$ is a Lie group and then $G$ itself is a Lie group by Lemma (3.2).

Assume that $H$ is general, and choose a compact normal subgroup $K$ of $H$ so that $H / K$ is a Lie group. Then $\varphi$ induces a dense embedding $\hat{\varphi}: H / K \rightarrow$ $G / \varphi(K)$. By what we have observed above, $\hat{\varphi}$ is an isomorphism, which implies that $\varphi$ is an isomorphism.

COROLLARY. Let $G$ be a connected locally compact group with its radical $R(G)$. If $S$ is a closed connected semisimple normal subgroup of $G$ such that $G / S$ is abelian, then $G=R(G) S$.

Proof. First we note that $\overline{R(G) S}=G$. To see this, consider the induced

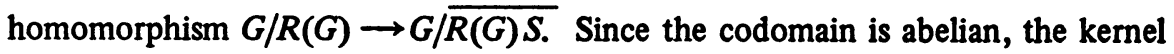
of this homomorphism contains the commutator subgroup of $G / R(G)$. But $G / R(G)$ is semisimple and hence its commutator subgroup is dense in $G / R(G)$ (3.1). Therefore this homomorphism is trivial and $G=\overline{R(G) S}$ foliows. Now the 
induced homomorphism $S / S \cap R(G) \rightarrow G / R(G)$ is a dense embedding of a semisimple group into another such group. Thus by the preceding proposition, this embedding is an isomorphism and $G=R(G) S$ as follows.

(3.4) Proposition 4. Let $G$ be a connected locally compact group. Then $G=R(G) \overline{G^{\prime}}$, where $\overline{G^{\prime}}$ denotes the closure on the commutator subgroup $G^{\prime}$ of $G$.

Proof. Let $H$ denote $\overline{G^{\prime}}$ and consider the radical $R(H)$ of $H$. Then $R(H)$ $\subset R(G)$ and $R(G) / R(H)$ is the radical of $G / R(H)$. Applying the above corollary to the semisimple subgroup $H / R(H)$ of $G / R(H)$, we get $G / R(H)=R(G) / R(H) \cdot$ $H / R(H)$, and hence $G=R(G) \cdot H$ follows.

(3.5) Let $G$ be a connected locally compact group and let $\left\{K_{\lambda}\right\}$ be a family of compact normal subgroups of $G$ so that each $G / K_{\lambda}$ is a Lie group and that $G=\lim G / K_{\lambda}$.

LEMMA. If the adjoint representation of each Lie group $G / K_{\lambda}$ is semisimple, then $R(G)=Z(G)_{0}$.

Proof. Let $G_{\lambda}$ denote the Lie algebra of $G / K_{\lambda}$. Then under the hypothesis each $G_{\lambda}$ is reductive (that is, the adjoint representation of $G_{\lambda}$ is semisimple). Thus the radical of $G_{\lambda}$ is central in $G_{\lambda}$, which implies that $R\left(G / K_{\lambda}\right)$ is central in $G / K_{\lambda}$. Since $R(G) K_{\lambda} / K_{\lambda}=R\left(G / K_{\lambda}\right)$ for each $\lambda$, it follows that $[R(G), G] \subset$ $\cap_{\lambda} K_{\lambda}=1$, proving that $R(G)$ is central in $G$. That $Z(G)_{0} \subset R(G)$ is clear.

REMARK. A group satisfying the hypothesis of the above lemma is termed as a proreductive group by Moskowitz [10], in which he obtained some of the important properties of such groups. Of course, our definition of reductivity of Lie groups requires a much stronger condition than his and every reductive group is certainly proreductive. However the lemma together with Proposition $4 \mathrm{im}$ plies the first part of the following.

Proposition 5. Let $G$ be a connected reductive group. Then

(i) $G=Z(G)_{0} \cdot \overline{G^{\prime}}$,

(ii) $G / \bar{G}^{\top}$ is compact.

Proof. (ii) follows directly from the fact that $\operatorname{Hom}(G, R)=0$ if $G$ is reductive.

4. The automorphism group of connected reductive groups.

(4.1) Let $G$ be a locally compact group and let $A(G)$ denote the group of all (bicontinuous) automorphisms of $G$. Then there is a natural topology on $A(G)$ under which this becomes a topological group. This topology, first introduced by Braconnier (J. Math. Pures Appl. 27 (1948)), is in general stronger than the so-called compact open topology. However when $G$ is connected (or more 
generally $G$ is compact modulo its identity component), these two topologies on $A(G)$ are the same (see [13]). Thus, in this case, a fundamental system of neighborhoods of 1 consists of the set $N(C, U)$ of all $\theta \in A(G)$ with $\theta(x) x^{-1} \in U$ for all $x \in C$, as $C$ and $U$ run over all compact subsets and all 1-neighborhoods of $G$, respectively. Throughout we will use $A_{0}(G)$ to denote the identity component of $\mathrm{A}(G)$. The following notation will be also used. For each $x \in G, I_{x}$ denotes the inner automorphism of $G$ that is induced by $x$. More generally, if $A$ is a subset of $G$, then $I_{G}(A)$ denotes $\left\{I_{x}: x \in A\right\}$. In the case $A=G$, we simply write $I(G)$ instead of $I_{G}(G)$.

(4.2) Theorem 2. Let $G$ be a connected reductive group. Then $A_{0}(G)$ is locally compact and $A_{0}(G)=I(G)$.

Proof. Let $K$ be a compact normal subgroup of $G$ so that $G / K$ is a Lie group and consider the map $\varphi_{*}: A_{0}(G) \rightarrow \operatorname{Hom}(G, G / K)$ which is induced by the projection $\varphi: G \rightarrow G / K$. By Theorem $1, I(G / K) \circ \varphi$ is the connected component of $\varphi$ in $\operatorname{Hom}(G, G / K)$. Since $\varphi_{*}$ sends the identity map to $\varphi$, it follows that $\operatorname{Im} \varphi_{*} \subseteq \operatorname{Int}(G / K) \cdot \varphi$. Thus if $\theta \in A_{0}(G)$, then there exists $y_{0} \in G$ such that $\varphi \cdot \theta(x)=\varphi\left(y_{0}\right) \varphi(x) \varphi\left(y_{0}\right)^{-1}$ for all $x \in G$. We show that $\theta$ is an inner automorphism.

Let $\left\{K_{\lambda}\right\}_{\lambda \in \Lambda}$ be a family of compact normal subgroups with each $K_{\lambda} \subset K$ such that $G / K_{\lambda}$ is a Lie group for each $\lambda \in \Lambda$ and that $G$ is a projective limit of $G / K_{\lambda}$. Thus, for each $\lambda \in \Lambda$, there exists $y_{\lambda} \in G$ such that for $x \in G, \theta(x)=$ $y_{\lambda} x y_{\lambda} \bmod K_{\lambda}$. Since each $K_{\lambda} \subseteq K$, we have $y_{\lambda} x y_{\lambda}^{-1}=y_{0} x y_{0}^{-1} \bmod K$, which implies that $\varphi\left(y_{\lambda}^{-1} y_{0}\right)$ is in the center of $G / K$. We prove that, for each $\lambda \in \Lambda$, there exists $a_{\lambda} \in Z(G)$ such that $\varphi\left(a_{\lambda}\right)=\varphi\left(y_{\lambda}^{-1} y_{0}\right)$. To do this, we first note that $G=K \cdot Z_{G}(K)$, where $Z_{G}(K)$ denotes the centralizer of $K$ in $G$ (see [7, Theorem 2, p. 515]). Thus we can find $a_{\lambda} \in Z_{G}(K)$ so that $\varphi\left(a_{\lambda}\right)=$ $\varphi\left(y_{\lambda}^{-1} y_{0}\right)$. Since $K$ is normal in $G$, so is $Z_{G}(K)$ and hence $\left[a_{\lambda}, x\right]=$ $a_{\lambda} x a_{\lambda}^{-1} x^{-1} \in K \cap Z_{G}(K)=Z(K)$.

If $x_{1}, x_{2} \in G$, then $\left[a_{\lambda}, x_{1} x_{2}\right]=\left[a_{\lambda}, x_{1}\right]\left[a_{\lambda}, x_{2}\right]$. (This follows from the fact that $Z(K)$ is in the center of $G$.) Thus we have a continuous homomorphism $x \rightarrow\left[a_{\lambda}, x\right]$ from $G$ into the abelian group $Z(K)$. Hence the kernel of this homomorphism contains $\overline{G^{\prime}}$ and $a_{\lambda}$ centralizes $\overline{G^{\prime}}$. Since $G$ is reductive, $G=Z(G) \overline{G^{\prime}}$ (Proposition 5). Hence $a_{\lambda}$ is central.

Now we have $\varphi\left(a_{\lambda}\right)=\varphi\left(y_{\lambda}^{-1} y_{0}\right)$, from which $y_{\lambda} a_{\lambda} \in y_{0} K$ for each $\lambda \in \Lambda$. Replacing $y_{\lambda}$ by $y_{\lambda} a_{\lambda}$ if necessary, we may assume that $y_{\lambda} \in y_{0} K$. Then the net $\left(y_{\lambda}\right)_{\lambda \in \Lambda}$ in the compact set $y_{0} K$ has a converging subnet $\left\{y_{\lambda_{j}}\right\}_{j}$ with $\lim _{j} y_{\lambda_{j}}=y$. Then $y x y^{-1}=\lim _{j}\left(y_{\lambda_{j}} x y_{\lambda_{j}}^{-1}\right)=\theta(x)$, proving that $\theta=I_{y}$.

It remains to show that $A_{0}(G)$ is locally compact. We first note that each $\theta \in A_{0}(G)$ induces an element $\hat{\theta} \in A_{0}(G / Z(G))$ and that $\theta \rightarrow \hat{\theta}$ defines a 
continuous isomorphism $A_{0}(G)=I(G) \cong A_{0}(G / Z(G))=I(G / Z(G))$. Since the domain $A_{0}(G)$ is connected, the local compactness of $A_{0}(G / Z(G))$ implies our assertion. Hence without loss of generality we may assume that $G$ has the trivial center and hence that $G$ is a semisimple group with trivial center (see Proposition 5 in §3). Let $K$ be the maximal compact connected normal subgroup of $G$. Then $G=K \cdot L$ where $L$ is the centralizer of $K$ in $G$ [7]. Since $G / K$ is a Lie group and since $K \cap L=1$, it follows that $L$ is a closed analytic subgroup of $G$. Consider the continuous homomorphism $\chi: A_{0}(G) \rightarrow A_{0}(K) \times A_{0}(L)$ which is defined by $\chi(\theta)=\left(\left.\theta\right|_{K},\left.\theta\right|_{L}\right), \theta \in A_{0}(G)$. Clearly $\chi$ is an injection. Since $A_{0}(G)$ $=I(G), A_{0}(K)=I(K)$ and $A_{0}(L)=I(L)$ by the first part of the theorem, it is also a surjection. Thus it follows from the connectedness of $A_{0}(G)$ that $\chi$ is an isomorphism of topological groups and $A_{0}(G)$ is locally compact.

\section{Nonconnected reductive groups and $A_{0}(G)$.}

(5.1) Proposition 6. Let $G$ be a reductive group such that $G / G_{0}$ is compact. Then $I_{G}\left(G_{0}\right)$ is dense in $A_{0}(G)$.

Proof. Let $\theta \in A_{0}(G)$ and consider a neighborhood $N(D, U)$ of 1 in $A(G)$, where $D$ is a compact subset and $U$ is a neighborhood of 1 in $G$. Since $G / G_{0}$ is compact, $U$ contains a compact normal subgroup $K$ of $G$ such that $G / K$ is a Lie group. Then the natural projection $\varphi: G \rightarrow G / K$ induces a continuous $\operatorname{map} \varphi_{*}: A_{0}(G) \rightarrow \operatorname{Hom}(G, G / K)$ with $\varphi_{*}(1)=\varphi$. By Theorem 1 in $\S 2$, we can find an element $h \in G_{0}$ such that $\varphi \cdot \theta(x)=\varphi \cdot I_{h}(x)$ for $x \in G$. Thus $I_{h}(x) \theta(x)^{-1} \in K \subset U$ for $x \in C$, proving that every neighborhood of $\theta$ contains an inner automorphism, and the assertion follows.

(5.2) Let $G$ be a reductive group such that $G / G_{0}$ is compact, and let A be the subgroup of $A_{0}(G)$ consisting of $\theta$ such that $\theta=1$ on $G_{0}$.

LEMmA. (i) $A$ is the center of $A_{0}(G)$.

(ii) $I_{G}\left(Z\left(G_{0}\right)\right)$ is dense in $A$.

(iii) $A_{0}(G)=A \cdot I_{G}\left(G_{0}\right)$.

Proof. Consider the restriction homomorphism $\chi: A_{0}(G) \rightarrow A_{0}\left(G_{0}\right)$. Clearly $\chi$ is continuous. Since $G / G_{0}$ is compact, $G_{0}$ is also reductive (by the corollary in (2.5)). Thus by Theorem 2 in $\S 4, A_{0}\left(G_{0}\right)=I\left(G_{0}\right)$ and this implies that $\chi$ is surjective. Since $A=\operatorname{Ker} \chi$, (iii) follows immediately.

To show (i), let $\theta$ be a central element of $A_{0}(G)$. Then $\chi(\theta)=\left.\theta\right|_{G_{0}}$ is central $A_{0}\left(G_{0}\right)$. But $A_{0}\left(G_{0}\right)=I\left(G_{0}\right)$ has the trivial center and $\theta \in \operatorname{Ker} \chi=A$. Conversely, let $\theta \in A$. If $h \in G_{0}, \theta\left(h x h^{-1}\right)=\theta(h) \theta(x) \theta(h)^{-1}=h \theta(x) h^{-1}$ for all $x \in G$, from which we see that $\theta \cdot I_{h}=I_{h} \cdot \theta, h \in G_{0}$. Since $I_{G}\left(G_{0}\right)$ is dense in $A_{0}(G)$ by Proposition 6 in (4.1), $\theta$ is central in $A_{0}(G)$. It remains to prove (ii). 
Let $\theta \in A$ and write $\theta=\lim _{\lambda} I_{h_{\lambda}}$ with $h_{\lambda} \in G_{0}$. For any $h \in G_{0}$, we denote by $I_{h}^{0}$ the inner automorphism of $G_{0}$ induced by $h$. Thus, with this notation, $I_{h}^{0}=\chi\left(I_{h}\right)$.

Now we have $1_{G_{0}}=\chi(\theta)=\lim _{\lambda} I_{h_{\lambda}}^{0}$. Since $A_{0}\left(G_{0}\right)=I\left(G_{0}\right)$ is locally compact, $G_{0} / Z\left(G_{0}\right) \cong I\left(G_{0}\right)$. Hence there exists a net $\left\{c_{\lambda}\right\}_{\lambda}$ with $c_{\lambda} \in Z\left(G_{0}\right)$ such that $\lim _{\lambda} h_{\lambda} c_{\lambda}^{-1}=1$ in $G_{0}$. Then we have $\lim _{\lambda} I_{h_{\lambda}} \cdot I_{c_{\lambda}}^{-1}=1_{G}$. This together with $\lim _{\lambda} I_{h_{\lambda}}=\theta$ implies that $\theta=\lim _{\lambda} I_{c_{\lambda}}$, proving that $\theta \in \overline{I_{G}(Z(G))}$.

(5.3) LEMMA. Let $G$ be a reductive group and let $V$ be a closed normal vector subgroup of $G$ such that $A_{0}(G / V)=I_{G / V}\left(G_{0} / V\right)$. Then $A_{0}(G)=$ $I_{G}\left(G_{0}\right)$.

Proof. By Proposition 5 in (3.5), $V$ is central in $G_{0}$ and by Proposition 6 in (5.1), every $\theta \in A_{0}(G)$ leaves every element of $V$ fixed. For $\theta \in A_{0}(G)$, let $\hat{\theta} \in A(G / V)$ be induced by $\theta$. Then $\theta \rightarrow \hat{\theta}$ defines a continuous homomorphism $A_{0}(G) \rightarrow A_{0}(G / V)$. By the hypothesis, we see that, for $\theta \in A_{0}(G)$, there exists $h \in G_{0}$ such that, for each $x \in G, \theta(x)=h x h^{-1} \bmod V$. Let $\alpha$ : $G \rightarrow V$ be defined by $\alpha(x)=h x h^{-1} \theta(x)^{-1} \in V, x \in G$. Then $\alpha \in Z^{1}(G, V)$, where we regard $V$ as a continuous $G$-module under the action $x \cdot v=$ $\theta(x) v \theta(x)^{-1}$ for $x \in G$ and $v \in V$. Since $G$ is reductive, the cocycle $\alpha$ is a coboundary. That is, there exists $v_{0} \in V$ so that $\alpha(x)=x \cdot v_{0}-v_{0}$. Writing multiplicatively, we see that $h x h^{-1}=v_{0}^{-1} \theta(x) v_{0}$ for all $x \in G$, which implies that $\theta=I_{v_{0} h} \in I_{G}\left(G_{0}\right)$.

(5.4) Now we are ready to prove the main result of this section.

THEOREM 3. Let $G$ be a locally compact group which is an extension of a connected semisimple group by a compact group. Then $A_{0}(G)=I_{G}\left(G_{0}\right)$.

Proof. Under the hypothesis, $G$ is reductive and $G / G_{0}$ is compact (Proposition 2 in (2.5)). There exists a compact subgroup $C$ of $G$ so that $G=G_{0} \cdot C$ [8, Theorem 2.13, p. 421]. Since $C$ acts on the connected abelian group $R(G)$ by conjugation, we can find a $C$-invariant closed vector subgroup $V$ of $R(G)$ such that $R(G) / V$ is compact (see [2, Satz IV, p. 163]). Since $V$ is central in $G_{0}$, it follows that $V$ is a normal subgroup of $G$. By Lemma (5.3), we may assume that $R(G)$ is compact. Let $H$ be a connected closed semisimple normal subgroup of $G$ such that $G / H$ is compact. Thus we can find closed normal subgroups $R_{1}$ and $S$ of $G$ such that both $R_{1}$ and $S$ contain $H$ and that $R_{1} / H$ and $S / H$ are the radical and the semisimple part of the compact group $G_{0} / H$, respectively. Clearly $G=R(G) \cdot S$ and $S$ is a connected semisimple group. Let $K$ be the maximal compact connected normal subgroup of $S$ and let $Z_{S}(K)$ denote the centralizer of $K$ in $S$. Then $S / K$ is a Lie group and $S=K \cdot Z_{S}(K), K \cap Z_{S}(K)$ 
$=Z(K)$ (see Iwasawa [6]). Let $S_{1}$ denote the identity component of $Z_{S}(K)$. Then $S_{1}$ is a closed normal subgroup of $G$ and $S=K S_{1}$. To see this, consider the (continuous) isomorphism $Z_{S}(K) / K \cdot S_{1} \cap Z_{S}(K) \cong G / K S_{1}$. As $Z_{S}(K)$ is $\sigma$ compact, this is an isomorphism of topological groups. Now since $K S_{1} \cap Z_{S}(K)$ contains $S_{1}, Z S(K) / K \cdot S_{1} \cap Z_{S}(K)$ is totally disconnected while $G / K S_{1}$ is connected. Hence $G / K S_{1}$ is trivial, which implies that $S=K S_{1}$. Since $S_{1} / S_{1} \cap K$ $\cong S / K$ is a Lie group, and since $S_{1} \cap K$ is contained in $Z_{S}(K) \cap K=Z(K)$, it follows from Lemma (3.2) that $S_{1}$ itself is a Lie group. Thus we have the decomposition $G_{0}=R(G) K S_{1}$ and $Z\left(G_{0}\right)=R(G) Z(K) Z\left(S_{1}\right)$. On the other hand, $A_{0}(G)=I_{G}\left(G_{0}\right)$ follows from the Lemma in (5.2) as soon as we show $\overline{I_{G}\left(Z\left(G_{0}\right)\right)}=I_{G}\left(Z\left(G_{0}\right)\right)$.

Since $R(G) Z(K)$ is compact, it is hence enough to show that $I_{G}\left(Z\left(S_{1}\right)\right)$ is closed in $A_{0}(G)$. Indeed, we show that $I_{G}\left(Z\left(S_{1}\right)\right)$ is discrete. To see this, let $C$ be as in the beginning of our proof and let $U$ be a 1-neighborhood of $G$ so that $Z\left(S_{1}\right) \cap U=1$. Then the neighborhood $N(C, U)$ meets $\overline{I_{G}\left(Z\left(S_{1}\right)\right)}$ trivially. In

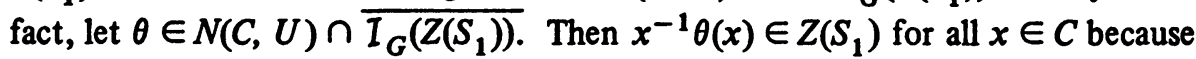
$\theta$ is in the closure of $I_{G}\left(Z\left(S_{1}\right)\right)$ and $Z\left(S_{1}\right)$ is a normal subgroup of $G$. Thus $x^{-1} \theta(x)$ $\in U \cap Z\left(S_{1}\right)=1$, which implies that $\theta=1$ on $C$. Clearly $\theta=1$ on $G_{0}$. Hence $\theta=$ 1 on $G=G_{0} C$, proving that $\overline{I_{G}\left(Z\left(S_{1}\right)\right)}$ is discrete.

REMARKS. 1. Regarding the reference to [2] (in our proof of Theorem 3), the proof there used an invariant integral on functions with values in a compact abelian group, which seems to require some further justification. For complete proofs, see Hofmann and Mostert [Splitting in topological groups, Mem. Amer. Math. Soc., No. 43 (1963), Theorem X] and also Grosser and Moskowitz [Compactness conditions in topological groups, J. Reine Angew. Math. 246 (1971), Theorem (1.1)].

2. Theorem 3 generalizes Iwasawa's Theorem as well as the classical result on semisimple analytic groups. See also [5, Theorem (4.3)] for a similar result for a certain type of nonconnected groups with semisimple identity component. For Lie groups, we have: If $G$ is a compactly generated reductive Lie group, then $A_{0}(G)=I_{G}\left(G_{0}\right)$. This is an immediate consequence of Theorem 1 of $\$ 2$.

\section{REFERENCES}

1. C. Chevalley, Théorie des groupes de Lie. Vol. 2: Groupes algébriques, Actualités Sci. Indust., no. 1152, Hermann, Paris, 1951. MR 14, 448.

2. H. Freudenthal, Ein Zerlegungssatz für im Kleinen kompakte Gruppen, Arch. Math. 15 (1964), 161-165. MR 29 \#3571.

3. M. Gotô, Linear representations of topological groups, Proc. Amer. Math. Soc. 1 (1950), 425-437. MR 12, 479.

4. - Dense embeddings of locally compact connected groups, Ann. of Math. (2) 61 (1955), 154-169. MR 16, 447. 
5. S. Grosser, O. Loos and M. Moskowitz, Über Automorphismengruppen lokal-kompakter Gruppen und Derivationen von Lie-Gruppen, Math. Z. 114 (1970), 321-339. MR 41 \#8575.

6. G. Hochschild and G. D. Mostow, Representations and representative functions of Lie groups, Ann. of Math. (2) 66 (1957), 495-542. MR 20 \#5248.

7. K. Iwasawa, On some types of topological groups, Ann. of Math. (2) 50 (1949), 507-558. MR 10, 679.

8. D H. Lee, Supplements for the identity component in locally compact groups, Math. Z. 104 (1968), 28-49. MR 36 \#6534.

9. - On deformations of homomorphisms of locally compact groups, Trans. Amer. Math. Soc. 191 (1974), 353-361.

10. M. Moskowitz, On pro-reductive groups, Proc. Cambridge Philos. Soc. 76 (1974), 401-406. MR 49 \#10818.

11. G. D. Mostow, Cohomology of topological groups and solvmanifolds, Ann. of Math. (2) 73 (1961), 20-48. MR 23 \#A2484.

12. W. T. van Est, Dense embeddings of Lie groups, Nederl. Akad. Wetensch. Proc. Ser. A 54 = Indag. Math. 13 (1951), 321-328. MR 13, 432.

13. S. P. Wang, The auromorphism group of a locally compact group, Duke Math. J. 36 (1969), 277-282. MR 39 \#1589.

OHIO 44106

DEPARTMENT OF MATHEMATICS, CASE WESTERN UNIVERSITY, CLEVELAND, 